\title{
Marco de Referencia de la Aplicación de Manufactura Esbelta en la Industria
}

\author{
A FRAMEWORK FOR THE IMPLEMENTATION OF LEAN MANUFACTURING IN THE INDUSTRY
}

\section{Jessica Tapia Coronado', Teresa Escobedo Portillo, Enrique Barrón López', Guillermina Martínez Moreno', Virginia Estebané Ortega'}

1. Universidad Autónoma de Ciudad Juárez, Chihuahua, México.

\section{RESUMEN}

Actualmente las empresas que aplican las herramientas de Manufactura Esbelta cometen el error de implementarlas de manera aislada para cubrir las necesidades de mejora a un corto plazo, por lo que obtienen beneficios limitados. Se presenta una revisión de literatura relacionada con la implementación de herramientas de Manufactura Esbelta en la Industria, tales como Takt Time, 5's, Ocho desperdicios "mudas", Control Visual, Células de Manufactura, a prueba de errores (Poka-Yoke), Nivelación de la producción (Heijunka), Automatización inteligente (Jidoka), Mejora continua (Kaizen), Kanban, Cambios rápidos de modelo (SMED), Mantenimiento total de la producción (TPM), Justo a tiempo (JIT) y Mapeo del flujo de valor (VSM), analizando su aplicación tanto individual como en conjunto. Se visualiza que las 5'S, el VSM, Kaizen, Kanban y TPM son las más utilizadas en el ramo Manufacturero con un 9,46\%, 8.1\%, 6,75\%, $5,4 \%$ y 4,05\% respectivamente, y SMED con un 4,05\% y JIT con un $6,76 \%$ en el sector Automotriz; caso contrario, las Células de Manufactura, Heijunka y Andon son las menos utilizadas (en 1,35\%).

(Tapia J, Escobedo T, Barrón E, Martínez G, Estebané V, 2017. Marco de Referencia de la Aplicación de Manufactura Esbelta en la Industria. Cienc Trab. Sep-Dic; 19 [60]: 171-178).

Palabras Clave: MANUFACTURA ESBELTA, MEJORA CONTINUA, INDUSTRIA.

\section{ABSTRACT}

Currently the industries that apply Len Manufacturing tools commit the mistake of implement them in insolation to meet short-term improvement needs, so that, they obtain limited benefits. A literature review present is related with the implementation of Lean Manufacturing tools in the industry, such as Takt Time, 5's, Eight wastes “mudas", Visual Control, Manufacturing Cells, mistake proofing (Poka-Yoke), Leveling production (Heijunka), Intelligent automation (Jidoka), Continuous improvement (Kaizen), Kanban, Quick changeover of model (SMED), Total productive maintenance (TPM), Just in time (JIT) and Value stream map (VSM), analyzing their application both individual and in assemblage. It is visualized that the 5 's, the VSM, Kaizen, Kanban and TPM are the most utilized tools in the Manufacturing branch with 9.46\%, 8.1\%, 6.75\%, 5.4\% and 4.05\% respectively, and SMED with 4.05\% and JIT with 6.76\% in the Automotive sector, otherwise, the Manufacturing Cells, Heijunka and Andon are the least used in 1.35\%.

Keywords: LEAN MANUFACTURING, CONTINUOUS IMPROVEMENT, INDUSTRY.

\section{INTRODUCCIÓN}

En cada empresa existen actividades, ya sean mucho o poco visibles, qufe generan pérdida de dinero, las cuales, de acuerdo a Womack et $\mathrm{al}^{1}$, son llamadas Muda (palabra japonesa que significa desperdicio). Como explica $\mathrm{Ohno}^{2}$, el desperdicio es el mal de todo proceso en una empresa, ya que involucra específicamente cualquier actividad humana que absorbe recursos, pero no crea valor tales como errores que requieren re trabajo, artículos para produc-

Correspondencia / Correspondence:

María Teresa Escobedo Portillo

Doctora en Planeación Estratégica y Dirección de Tecnología

Av. Plutarco Elias Calles 1210, Código Postal 32310

Tel.: (+52) 6884800 ext. 5458

e-mails: mayteesc@gmail.com • mtescobe@uacj.mx

Recibido: 15 de Junio de 2017 / Aceptado: 24 de Agosto de 2017 ción no requeridos que se acumulan en inventarios, etapas dentro del proceso que no son necesarias, movimiento de empleados y transportes de materia prima de un lugar a otro sin propósito; son algunos ejemplos de actividades que causan gastos innecesarios a la empresa. ${ }^{3}$

Para poder responder a este reto se deben desarrollar estrategias de operaciones que se aproximen a ayudar en reducir los costos de operación y al mismo tiempo eliminar desperdicios para alcanzar la calidad del producto final, y esto se logra usando la Manufactura Esbelta y eliminando las mudas. ${ }^{4}$

Sin embargo, se tiene que desarrollar un pensamiento que provenga de enfoques esbeltos para la implantación de herramientas, técnicas y metodologías para la manufactura que ayudan a lograr los beneficios de producir según los procesos lo requieran, en donde el éxito se deberá a las decisiones financieras y organizacionales tomadas por la gerencia ${ }^{5}$; es por eso que la Manufactura Esbelta es usada para cambiar a las empresas y adaptarlas a las necesidades de los clientes.

Puesto que existen diferentes herramientas dentro de la Manufactura Esbelta en donde se persigue fabricar productos de calidad con valor agregado para el cliente ${ }^{6}$, lo que se busca es la 
integración completa de todas esas técnicas para lograr que su implantación sea estable y perpetua, donde se obtengan las ganancias deseadas por la gerencia refiriéndose a costos, y logre un cambio de mentalidad de trabajo en todos los asociados llegando a un involucramiento altamente efectivo que genere un sentido de pertenencia hacia el proceso ${ }^{7} \mathrm{y}$, por lo tanto, hacia la empresa, evitando con esto regresar a la antiguas prácticas de fabricación. El objetivo de esta revisión literaria es exponer el uso de las herramientas de manufactura esbelta para la mejora de sistemas y procesos en la actualidad, dándose a conocer las tendencias de uso dentro de la industria, su aplicación en conjunto con las técnicas de la manufactura esbelta y su combinación con metodologías de otras áreas.

\section{MANUFACTURA ESBELTA}

La estrategia de mejora continua en la producción compuesta por un conjunto de herramientas administrativas cuyo objetivo es ayudar a eliminar operaciones que no le agregan valor al producto y a los procesos, reducen o eliminan desperdicios para mejorar las operaciones bajo un ambiente de respeto por el trabajador, se conoce como Manufactura Esbelta. ${ }^{8}$

Es importante saber que el sistema de Manufactura Esbelta está compuesto por varios subsistemas (herramientas) ${ }^{9}$, y que éstos son usados para reducir y eliminar el desperdicio en las empresas. ${ }^{10} \mathrm{La}$ manera para describir la Manufactura Esbelta, herramientas y aplicaciones, es definiendo las generalidades de cada una de ellas. En los siguientes párrafos se muestra la información general de las diferentes herramientas.

\section{Herramientas de Manufactura Esbelta}

\section{Takt Time}

La palabra Takt viene del alemán 'taktzeit' que significa ritmo, compás; hablando en términos para la ingeniería industrial es tiempo de ciclo, pero a diferencia del tiempo de ciclo que se conoce, este mide el ritmo de trabajo en una planta de manufactura. En lo que respecta a la producción esbelta, el TAKT TIME es el ritmo en que los productos deben ser completados o finalizados para satisfacer las necesidades de la demanda.2

Como ejemplo del beneficio de utilizar esta herramienta, se logró reducir el 2\% en desperdicio de jugo de mango dentro de una Industria Alimenticia, usado como indicador de resultados obtenidos y pre-requisito para la aplicación de otras técnicas de Manufactura Esbelta, obteniendo un total del 34\% de reducción de desperdicios para esa línea de producción. ${ }^{11}$

\section{5'S}

Esta metodología está orientada a un sistema de limpieza, organización y estandarización del área de trabajo; es un método para involucrar a la gente y contribuir al cambio de cultura laboral. ${ }^{12}$ Fue desarrollada por Hiroyuki Hirano para mejorar la industria y prepararla para recibir otras filosofías para que sea de clase mundial y sus procesos de excelencia. ${ }^{13}$ El método de las 5's es un camino efectivo para crear compromiso en los operarios y contribuir a la cultura de cambio. ${ }^{14}$

La herramienta de las 5's es de origen japonés, y se denomina de tal manera porque la primera letra del nombre de cada una de sus etapas es la letra (s). Esta técnica se compone de cinco principios fundamentales, los cuales son: Seiri que significa clasificación u organización, Seiton que es orden, Seiso significa limpieza, Seiketsu que es estandarización y, por último, Shitsuke que significa disciplina. ${ }^{15}$

En su aplicación se ha logrado adoptar el cumplimiento de esta técnica en un 65\%, como en el caso de la Industria de Confecciones, diseño, corte y costura en pieles y telas, en el área de Cartagena, Colombia, donde se usó para toda la empresa, desde guardar o vender máquinas que no estaban en uso hasta generar la cultura de limpieza en lo operarios.12 Un segundo caso es el presentado por Wilches et $\mathrm{al}^{14}$, en una empresa de fabricación de sillas para oficina donde se realizó la capacitación de todo el personal del área de formado de los tubos metálicos en la técnica de 5'S, obteniendo un aumento del 13\% en la calidad del formado.

\section{Ocho desperdicios (Mudas)}

Desperdicio es todo derroche o desaprovechamiento de los recursos y talento con los que cuenta una organización, tales como materiales, maquinaria y equipo, tiempo, espacio, competencias, talento humano, entre otros. El uso productivo de los recursos conduce a la reducción del desperdicio y a la conservación de los mismos, ya sean escasos o más caros. 0 hno $^{2}$ identificó siete tipos de desperdicios a los que posteriormente Womack y Jones ${ }^{16}$ agregaron uno más para tener los ochos desperdicios básicos.

Las mudas identificadas son las siguientes: la primera muda es la sobreproducción, descrita por procesar artículos anticipándose al requerimiento o en mayor cantidad que la solicitada por el cliente, a lo que se considera el principal desperdicio o la causa de la mayoría de los otros desperdicios. ${ }^{17}$

La segunda muda es el exceso de inventario, la cual consiste en el excesivo almacenamiento de materia prima o materiales, producto en proceso y producto terminado. ${ }^{18}$

La tercera muda se refiere a los retrasos, esperas y paros, la cual implica tener al personal esperando por información, instrucciones de trabajo, materiales, piezas o herramientas necesarias para realizar su labor; clientes o visitantes esperando a ser atendidos; piezas esperando para continuar su procesamiento; maquinaria parada por averías, entre otros. ${ }^{19}$

La cuarta muda es el transporte y envíos, la cual consiste en mover trabajo en proceso de un lado a otro, incluso cuando se recorren distancias cortas; también incluye el movimiento de materiales, partes o producto terminado hacia o desde el almacén, otras áreas o procesos. ${ }^{20}$

La quinta muda son los desplazamientos y movimientos innecesarios, que se refiere a cualquier movimiento físico o desplazamiento que el personal realice y que no agregue valor al producto o servicio, por ejemplo, cuando las personas deben bajar y subir documentos, desplazarse para buscar materiales, entre otros. ${ }^{21}$

La sexta muda se refiere al sobre-procesamiento y actividades que no agregan valor al cliente, donde se realizan procedimientos innecesarios tales como contar, acomodar, inspeccionar, revisar o duplicar procesos. También se incluye utilizar herramienta 0 equipo inapropiado, desarrollar características o funciones en los productos que no son valoradas por los clientes. ${ }^{22}$

La séptima muda es la de rechazos, fallos y defectos, la cual está dada por la corrección de errores y re-trabajo derivado de la identificación de no conformidades o por devoluciones del cliente, destruir o re-procesar productos que no reúnen las condiciones óptimas de calidad. ${ }^{9}$

La octava muda es el talento poco utilizado, en el cual se desapro- 
vecha la creatividad e inteligencia de los colaboradores, sus competencias y potencial para eliminar desperdicios, mejorar la productividad y resolver los problemas de calidad e innovación. ${ }^{23}$ Casos de estudio muestran los porcentajes de actividades que tienen desperdicios y, por lo tanto, no agregan valor al producto, tal como el realizado por Indrawati y Ridwansyah ${ }^{24}$, en la industria Iron Ores. Analizando el proceso de secado del mineral se encontró $33.67 \%$ de valor no agregado a las operaciones, ayudándoles a tomar la decisión de atacar los desperdicios encontrados. Un segundo caso es el de Juthamas et $\mathrm{al}^{22}$, donde aplican esta técnica en el proceso de estampado de hoja de metal, donde se redujo las actividades que no agregan valor en un 66.53\%.

\section{Control Visual, gestión a primera vista}

La colocación a simple vista de todas las herramientas, partes, actividades e indicadores del rendimiento del sistema de producción, para que el estado del sistema pueda ser conocido y entendido por cualquiera en un solo golpe de vista, se logra mediante la implementación de controles visuales que forman parte de la técnica llamada Fabrica Visual, también conocida con el término de transparencia ${ }^{25}$, la cual, dentro del sistema productivo puede ayudar a hacer visibles los problemas o debilidades del sistema, permitiendo que se tomen las medidas adecuadas de manera instantánea. $^{9}$

De manera general, el control visual se define como la información justo a tiempo que asegura una ejecución rápida y apropiada de las operaciones y los procesos. ${ }^{26}$ Un ejemplo de la vida diaria son las señales de tráfico, las cuales no requieren ser estudiadas, sino que su significado se hace evidente al instante.

Dentro de los elementos básicos de la gestión a primera vista está el sistema Andon, que muestra la localización y la naturaleza de los problemas resaltándolos de manera evidente ${ }^{27}$. Otro elemento es la hoja de estandarización de la tarea, que tiene como fin determinar inequívocamente las fallas del operario de manera que un observador externo pueda saber a simple vista si se está realizando correctamente la operación. ${ }^{28}$

Caso de estudio presentado por Muhammad et $\mathrm{al}^{29}$, donde se aplicó esta técnica al área de administración de la energía en la industria, se mostró un mejoramiento vital en el ahorro de energía por encima del 57\% y el uso del espacio en un 22\%; y, en otro caso, aplicado en el proceso de recolección de datos en sensores semicontinuos, se obtuvo un decremento del 15.35\% en problemas de disponibilidad de datos. ${ }^{30}$

\section{Células de Manufactura}

Una célula de manufactura agrupa todas las operaciones necesarias para producir y mantener flujos de producción continuos y las actividades indispensables para producir un componente o el subensamble acomodándolos de manera cercana unas de otras, lo cual permite la retroalimentación entre operadores ante problemas de calidad que se puedan presentar. ${ }^{2}$

Por definición, una célula de manufactura es un grupo de máquinas o procesos agrupados y dedicados a la fabricación de una familia de partes, donde estas son similares en sus requerimientos de proceso, tales como operaciones, tolerancias, utilización del herramental para máquinas, entre otros. ${ }^{5}$ Las llamadas células en forma de U permiten tener la ventaja de controlar el flujo pieza a pieza, y dar la facilidad del mantenimiento a la maquinaria. ${ }^{1}$

Stefan et $\mathrm{al}^{31}$ aplicaron esta técnica en el área de maquinado con
CNC, donde se analizó mediante un acomodo celular de la maquinaria y evaluándolo mediante control estadístico se obtuvo el resultado de detección de variación de los procesos de un 1.5\% a 6.3\%, concluyendo que esta técnica es responsiva a la flexibilidad del trabajo.

\section{Poka-Yoke}

El termino Poka-Yoke fue aplicado por Shigeo Shingo en 1986, dentro de los procesos industriales diseñados para prevenir errores humanos en los productos, llamándola a prueba de errores, dado por las palabras japonesas Poka que es evitar y Yokeru que significa error inadvertido. Rediseñó un proceso en el cual los trabajadores, mientras ensamblaban un pequeño interruptor, muy a menudo olvidaban insertar los cables requeridos por debajo de uno de los botones del mismo. En el proceso rediseñado, el trabajador podía realizar esta tarea en solo dos pasos. ${ }^{26}$

$\mathrm{Su}$ objetivo es evitar que se cometan errores, tanto a la hora de colocar mal una pieza, de ponerla en un lugar que no le corresponde o no ponerla cuando es necesario. ${ }^{32}$ Esto se consigue, por ejemplo, mediante el diseño de la forma de la pieza, la cual no permite que se coloque de ninguna otra manera que no sea la correcta, como sucede con los enchufes eléctricos de las paredes de cualquier hogar.

Esta técnica aplicada puede representar grandes ahorros económicos para las empresas, como es el caso de la empresa Solomoflex, donde se diseñó un sistema semi-automático usando Poka-Yoke para el proceso de fabricación de tijera 45D de la motocicleta Yamaha, obteniendo un ahorro por jornada laboral de $\$ 44.654$ dólares por cada 250 tijeras. $^{33}$

\section{Jidoka, Automatización inteligente}

Según indica $\mathrm{Ohno}^{2}$, en sus primeros años las máquinas tenían una gran capacidad productiva a expensas de que cualquier pequeña anomalía podía dañarlas de manera relativamente fácil. Cuando esto sucedia, se producian en poco tiempo decenas y centenas de piezas defectuosas. Con una máquina de este tipo la producción masiva de piezas no conformes era inevitable, al no tener ningún tipo de mecanismo para detectar cuándo sucedía algún problema. ${ }^{34}$ Por lo anterior, la empresa Toyota hizo énfasis en dicha herramienta, ya que las máquinas se diseñan para prevenir problemas de manera autónoma por delante de la automatización simple. La idea provino del sistema a prueba de errores creado por Sakichi Toyoda en 1896 para telares mecánicos, conocido por el término japonés Poka-Yoke. Dicha máquina, al tener un sistema que podía distinguir entre la condición normal de trabajo y una anormal, se detenía cuando detectaba un problema, evitando fabricar productos defectuosos, siendo esta una manera de dotar de inteligencia humana a las máquinas. ${ }^{35}$

El parar la máquina cuando falla hace que todos se fijen en ella, por lo cual sólo se puede mejorar un problema cuando se conoce a profundidad qué lo causa; con esta idea se estableció la regla de que incluso en operaciones de tipo manual, los trabajadores debian parar la línea pulsando el botón correspondiente cuando surgiera un contratiempo que alterara la producción, para ello se debía tener un conocimiento preciso de todo posible problema que pudiera surgir. $^{2}$

En 1997, por definición del exvicepresidente Alex Warren, de Toyota Motor Corporation en Kentucky, el sistema Jidoka dota de poder y responsabilidad a las personas encargadas del proceso, donde se les da la posibilidad de presionar un botón o tirar de una 
cuerda llamada Andon (término japonés que significa "lámpara", y se refiere a un sistema utilizado para alertar en un proceso de producción la presencia de problemas que hace que la línea pare; todo miembro del equipo tiene la responsabilidad de realizar esta actividad si algo se sale de la normalidad del sistema). ${ }^{36}$ En el caso de las máquinas, se le añaden dispositivos que detectan anormalidades y paran automáticamente la producción, entrando el equipo de trabajo con el conocimiento para resolver en el mínimo del tiempo el problema presentado. ${ }^{26}$ Esta herramienta hace que las personas sientan el compromiso y autoridad de mejorar la calidad en la producción, además de sentirse importantes dentro de la empresa.

\section{Kaizen}

Kaizen es un término japonés que significa "cambio a mejor" o "mejora" y es habitual mente traducido como mejora continua, y permite que todos los miembros de la organización estén buscando continuamente formas de mejorar cada aspecto de la misma, y que todos están de acuerdo y apoyan este tipo de mentalidad. Esta técnica requiere también una visión clara a la hora de saber qué se quiere conseguir y hacia dónde se quiere llegar. ${ }^{37}$

Esta herramienta no es simplemente un paquete de mejoras desarrolladas e implementadas por expertos en la organización, sino que implica a todos, alimentándose de la experiencia y el conocimiento de cada uno. ${ }^{38}$ La mejora continua solo puede ocurrir cuando un proceso es estable y está estandarizado. ${ }^{39}$

La raíz del Kaizen es una actitud de introspección y autocrítica, una manera de pensar por parte de todos los componentes de la empresa, con un deseo arrollador de mejorar. ${ }^{40}$ En occidente se tiende a ver la crítica y la admisión de los errores como algo negativo o como un signo de debilidad9; en Toyota, por lo contrario, no hay mayor signo de fuerza que cuando alguien puede exponer abiertamente las cosas que no ha hecho bien, asumir la responsabilidad por sus errores y proponer medidas para asegurar que no vuelva a suceder el error. ${ }^{41}$

\section{Kanban}

Es una forma simple y sencilla de comunicación que hace que todo se encuentre en el sitio adecuado cuando se necesita; en la mayoría de los casos, es una pequeña tarjeta de papel insertada dentro de un recipiente rectangular de plástico en donde se escribe toda la información acerca de cuantas piezas se necesitan, cuándo y de qué manera, siendo éste la base del sistema de jalón dentro de los sistemas de producción esbeltos. ${ }^{42}$

Ohno ${ }^{2}$ instauró el Kanban como un supermercado, utilizándolo después de que el cliente realizase su adquisición, para indicar al departamento de compras cuáles son los artículos que se habrían de reponer. La finalidad de usar esta técnica es para conseguir que los movimientos en la planta de producción se sistematicen. ${ }^{43} \mathrm{El}$ Kanban es una manera de obtener una producción Justo a Tiempo, convirtiéndose en el sistema central de la producción que controla el flujo de los productos. ${ }^{44}$ Un caso de aplicación es el de la producción de café en Tailandia donde se redujo el número de operarios en un 13\% en las actividades exclusivas de transporte de materiales. ${ }^{45}$

Sus funciones son proveer información sobre la adquisición y transporte de los materiales, donde el proceso anterior toma el número de piezas indicado por el proceso inmediatamente posterior, proveer información acerca de la producción, en la cual el proceso anterior produce las piezas en la cantidad y la secuencia indicadas por el Kanban ${ }^{46}$ y prevenir la sobreproducción y el transporte excesivo, donde ninguna pieza se fabrica o se transporta si no existe un Kanban o tarjeta de pedido que lo justifique. ${ }^{2}$

También sirve como orden de producción adjunta a los materiales o piezas, previene la fabricación de piezas defectuosas mediante la identificación del proceso del que procede el defecto, evitando que lleguen a los procesos subsiguientes, lo que resulta en una producción libre de errores. ${ }^{47}$ Para reducir el tiempo de servicio en las consultas con el especialista en un hospital, se aplicó esta herramienta obteniendo un 67\%, generando valor para el cliente. ${ }^{48}$

SMED, Cambios rápidos de modelo.

Son las siglas de Single-Minute Exchange of Die, que en español se refiere a la técnica por la cual es posible modificar la configuración de una máquina en menos de 10 minutos o incluso segundos. ${ }^{37}$ Es importante porque una máquina con un tiempo de configuración elevado debe disponer de un exceso de capacidad para compensar dicho tiempo; por lo tanto, se debe acumular inventario para proveer de piezas a las siguientes etapas de la producción. ${ }^{2}$ Toda reducción del tiempo de configuración de la maquinaria reduce dos tipos de desperdicio: el exceso de capacidad y la sobreproducción. ${ }^{49}$

Este sistema elimina la producción en grandes lotes; su procedimiento es sencillo y se basa en tres etapas, la primera separa la configuración interna de la externa, la segunda convierte la configuración interna en externa y la tercera reestructura la configuración del cambio de modelo en el proceso. ${ }^{50}$

Cuando se aplica el SMED se utiliza un diagrama de Gantt mostrando todos los pasos de la configuración, o bien, se graba en video el proceso para poder estudiarlo; después de reunir a los encargados de la configuración para clasificar cada paso de las actividades tanto internas como externas, lo siguiente es eliminar los pasos innecesarios y simplificar lo que se pueda, para posteriormente convertir la mayor cantidad posible de pasos dentro de la configuración interna en configuración externa, logrando realizar el cambio de modelo cuando la máquina aún está encendida y evitar lo menos posible que esté parada. ${ }^{51}$

\section{Heijunka, Nivelación de la producción}

La nivelación del plan de producción tanto en volumen como en combinación de productos es un requisito necesario para el funcionamiento del sistema Justo a Tiempo y para la eliminación de desperdicios. ${ }^{10}$ La idea es nivelar el programa de producción secuenciando los pedidos según un patrón repetitivo que haga que la producción diaria media sea similar entre los distintos días de la semana, de manera que se puedan realizar modificaciones en la producción que correspondan con los pedidos a largo plazo, amortiguando los cambios suavemente. ${ }^{52}$

Esta herramienta es un elemento clave para lograr el flujo y la nivelación en la producción ${ }^{9}$, utilizando la técnica SMED, la cual hace posible cambiar la especificación de las máquinas en muy poco tiempo fabricando productos diferentes de manera consecutiva y utilizando la misma línea de montaje, donde los procesos están diseñados para permitir que los productos puedan ser cambiados fácilmente, produciendo lo que se necesita cuando se necesita; un caso claro de esta técnica es el del proceso productivo de fragancias en la empresa Disaromas S.A., donde se realizó la capacitación del personal para utilizarlo dentro de la mejora de nivelación de la producción, reduciendo en un 15\% la acumulación de inventario entre las estaciones de vaciado. ${ }^{53}$ 


\section{TPM, Mantenimiento productivo total}

Es una filosofía diseñada para integrar el mantenimiento del equipo en el proceso de fabricación. Es un sistema que mantiene y mejora la integridad de los sistemas de producción y de calidad a través de las máquinas, equipos y procesos que agreguen valor al producto. ${ }^{54}$ Este se centra en mantener todo el equipo en perfectas condiciones para evitar averías y retrasos, así como pequeñas paradas y defectos durante el proceso productivo proporcionando un entorno de trabajo seguro ${ }^{55}$ y una visión integral en toda la empresa para la gestión del mantenimiento, que puede dividirse en elementos a corto y largo plazo.

A largo plazo, los esfuerzos se centran en el diseño de nuevos equipos y la eliminación de tiempos muertos donde se requiere la participación de las diferentes áreas de la organización. A corto plazo, las actividades de TPM incluyen programas de mantenimiento autónomo y planeado para los departamentos de producción y mantenimiento. ${ }^{56}$

El objetivo de TPM es mejorar continuamente la disponibilidad y evitar la degradación de los equipos logrando la máxima eficacia, requiriendo de un fuerte apoyo de gestión, así como el uso continuo de los equipos de trabajo y las actividades de grupos pequeños para lograr mejoras. ${ }^{57}$ En el caso de Arslankayaa y Atayb58, lo aplicaron para agilizar la respuesta a los mantenimientos solicitados por la empresa y evitar incurrir en gastos innecesarios, donde se obtuvo un 75\% de aumento en el flujo de las órdenes de mantenimiento y acomodo de las partes solicitadas.

VSM, Mapa de flujo de valor.

Es importante gestionar la totalidad del flujo de valor para cada producto $^{59}$, para entender dónde se producen los desperdicios, en lugar de fijarse en actividades ${ }^{60}$ o procesos de manera aislada. ${ }^{37}$ Por lo anterior, debe crearse un mapa de flujo de valor donde se identifique cada una de las acciones requeridas para diseñar y fabricar un determinado producto, de manera que se puedan clasificar cada una de ellas en tres categorías. La primera son las actividades que crean valor desde el punto de vista del cliente; la segunda, aquellas que no crean valor desde el punto de vista del cliente, pero que son necesarias para llevar a cabo la producción, por lo tanto, no pueden ser eliminadas, pero sí pueden ser mejoradas a fin de reducir el tiempo que se les invierte; y la tercera y última, la actividades que no crean valor desde el punto de vista del cliente, y que son innecesarias, y estas puedan ser eliminadas inmediatamente. ${ }^{61}$

De los casos existentes donde se aplica esta técnica, se ha obtenido una reducción de tiempo de producción de turrón empaquetado del 83\% dentro de la industria alimenticia de Uruguay ${ }^{62}$, y también una reducción de personal en el área de cocina de un hospital en Dinamarca del $24 \%{ }^{63}$; otro de los casos es el presentado por Verma y Sharma64, donde aplican esta herramienta en el proceso de maquinado en CNC de un producto y cómo lograr ahorrar energía equivalente a más del 62\% al año.

\section{JIT, Justo a tiempo.}

Ohno ${ }^{2}$ desarrolló una nueva manera de coordinar el flujo de componentes a través de la cadena de suministro en el día a día con el sistema justo a tiempo, que se ha hecho posible gracias al sistema Kanban. La idea fue convertir un gran grupo de suministradores y de componentes en una gran máquina, como la planta Highland Park de Ford, dictando que las piezas sólo serían producidas para satisfacer la demanda inmediata del siguiente paso del proceso.
El mecanismo se llevaba a cabo a través del sistema Kanban, donde había contenedores que llevaban las piezas al siguiente paso; según se vaciaba cada contenedor, se enviaba de nuevo hacia el paso anterior, y esto suponía automáticamente una señal para que se fabricaran más piezas. ${ }^{15}$ Lo anterior era complicado de implementar en la práctica debido a que eliminaba todo el inventario y significaba que, si una sola pieza del sistema de producción fallaba, todo el sistema se vería paralizado, pero lo que se logró fue que se deshicieran todas las redes de seguridad y hacer que cada trabajador tuviera que concentrarse en prever los problemas antes de que fueran suficientemente graves como para tener que parar toda la producción. ${ }^{26}$

Este sistema consiste en hacer llegar las partes necesarias para el ensamblado a la línea en el momento justo que se necesitan y en la cantidad justa requerida ${ }^{9}$, donde puede reducir hasta un 63\% los tiempos de entrega, como en el caso del proceso de embotellado de vino en una empresa de España ${ }^{65}$ y en el caso de un proveedor de partes automotrices en la reducción del 45\% del inventario en espera de entrega a los diversos clientes. ${ }^{66}$

\section{Análisis de datos obtenidos.}

En este apartado se exponen gráficamente los resultados obtenidos de la revisión exhaustiva de la literatura del ramo con casos aplicados en el sector industrial usando las herramientas de Manufactura Esbelta, así como aquellas que son más y menos utilizadas por cada sector.

Figura 1.

Porcentaje por sector del uso de las herramientas de Manufactura Esbelta.

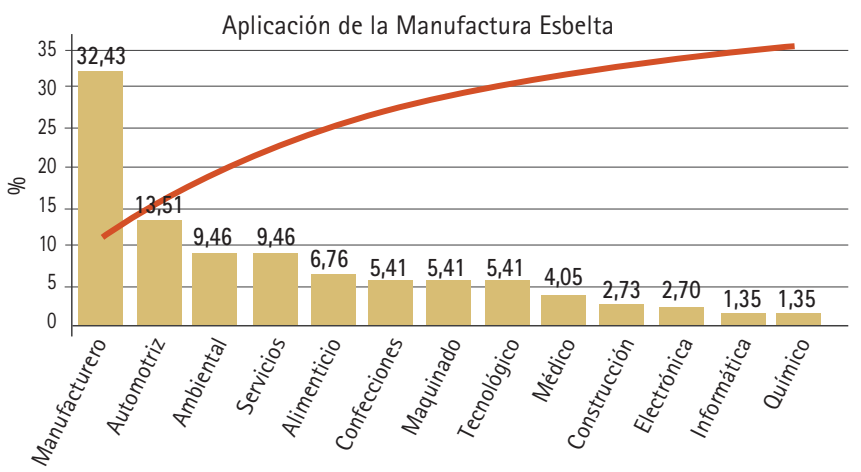

En este gráfico se muestra la tendencia de uso de las técnicas de la Manufactura Esbelta para los sectores industriales, apuntando que el sector con mayor aplicación es el Manufacturero con un 32,43\%, entendiéndose todas aquellas empresas de inyección de plásticos, ensamble componentes, neumáticos, pinturas, entre otros.

Figura 2.

Herramientas de Manufactura Esbelta aplicadas en la Industria.

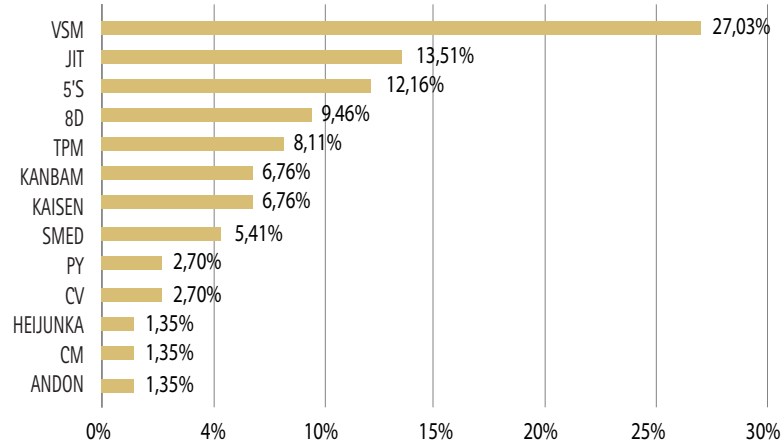

Fuente: Elaboración propia basada en autores de referencia. 
Se observa en la figura anterior cuáles son las herramientas mayormente aplicadas en la Industria, encontrando que VSM (Mapa de flujo de valor), sobresale en frecuencia de uso sobre las demás herramientas en un 27.03\%. Las herramientas Kanban y Kaizen tienen el mismo factor de aplicación con el 6,76\% y las de menor uso con 1,35\% son Células de Manufactura, Andon y Heijunka.

\section{Figura 3.}

Uso de la Manufactura Esbelta con otras metodologías.

Manufactura Esbelta y otras Metodologías

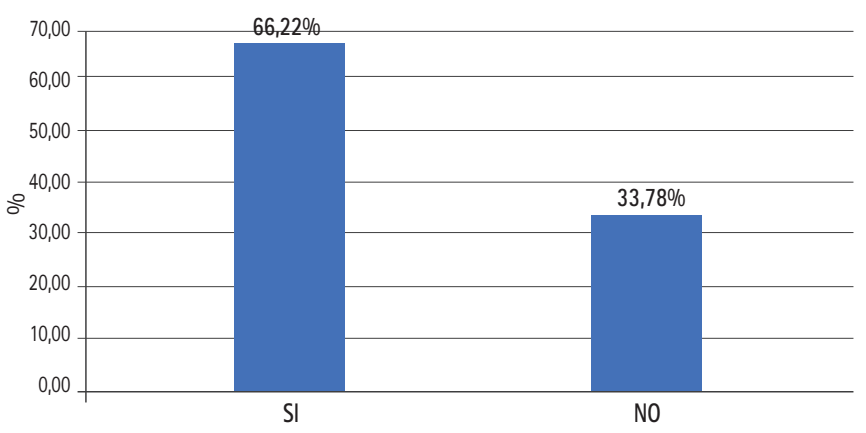

Fuente: Elaboración propia basada en autores de referencia.

Al investigar la aplicación de las herramientas de la Manufactura Esbelta, se percibe que algunas de ellas se combinan con otras Metodologías (Figura 3), tales como 5's, Kanban, VSM, TPM, SMED y JIT en un 66,22\% de los casos.

Existe una secuencia de aplicación de las herramientas de Manufactura Esbelta para un proceso (Figura 4), evitando su uso de manera aislada ${ }^{67}$. El 43,24\% de los procesos de fabricación, conjunta dos o más de éstas, existiendo una lógica de implementación para cada una de ellas, así como una serie de prerrequisitos que dependen del conocimiento que se tenga acerca de la metodología esbelta.

\section{CONCLUSIONES}

En la literatura del ramo se observa un enfoque en la aplicación de las diversas herramientas de la Manufactura Esbelta dentro de la industria, en la que se toman de manera aislada las herramientas que mejor les convenga, obteniendo así beneficios a corto plazo y olvidando mantenerlos a largo plazo. También se perciben metodologías para su implementación, que parecen ser muy eficaces, pero faltas de un sustento que rompa paradigmas que han perdurado desde la era de la producción en masa.

$\mathrm{Al}$ analizar la revisión de literatura se obtuvo que la herramienta VSM es la más aplicada en el sector industrial con un 27,03\% de ocurrencia, como lo demuestran Engelund et $\mathrm{al}^{63}$, Zahraee et al61 y Verma y Sharma ${ }^{64}$ en los estudios realizados tanto en la industria automotriz, ambiental y en la alimenticia. La herramienta de Justo a Tiempo con 13,51\% es aplicada mayormente en el sector alimenticio y automotriz, ya que se encuentran investigaciones como la de Jiménez et $\mathrm{al}^{65} \mathrm{y}$ Wang et $\mathrm{al}^{66}$, que la utilizó para el proceso de embotellado de vino en España y para la proveeduría de partes automotrices, respectivamente.

Arrieta $^{12}$ y Wilches et $\mathrm{al}^{14}$ utilizaron las 5's para la industria de confecciones para mejorar las áreas de diseño, corte y costura en
Figura 4.

Combinación de herramientas de Manufactura Esbelta.

Integración de herramientas de Manufactura Esbelta

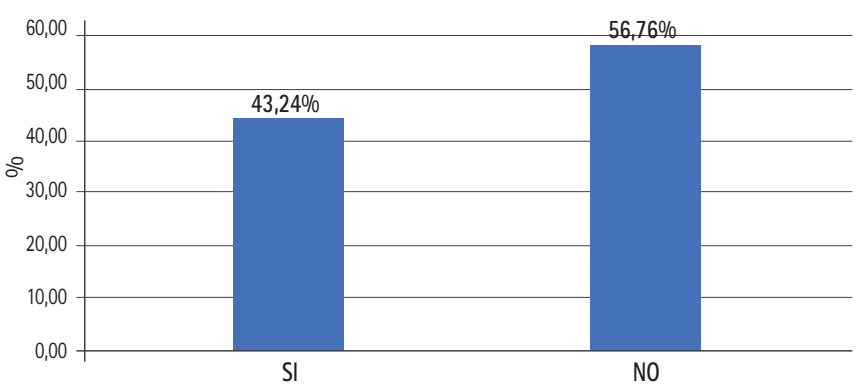

Fuente: Elaboración propia basada en autores de referencia.

un 65\% de productividad, y en una empresa de elaboración de sillas para oficina dentro el área de formado de tubos metálicos, obteniendo 13\% de aumento de la calidad en el producto final, respectivamente. Se observa que esta técnica tiene $12,16 \%$ de aplicación.

De los casos relacionados a la identificación de mudas, estos representan el 9,46\% de uso. Con esta técnica se logró identificar en una industria de tratamiento de minerales el 33,67\% de valor no agregado a las operaciones de secado. ${ }^{24}$ Un segundo caso donde se aplicó esta técnica fue en el proceso de estampado en hojas de metal, en el cual se redujo un 66,53\% las actividades que representaban un desperdicio para la producción. ${ }^{22}$

Para aumentar el porcentaje de flujo de órdenes de mantenimiento a las máquinas dentro de una industria de producción de lácteos Arslankayaa y Atayb ${ }^{58}$ utilizaron el TPM, teniendo como resultado el 75\%. Se observa de acuerdo a la Figura 2 que esta herramienta tiene un 8,11\% de aplicación.

Para el Kanban y Kaizen se tiene 6,76\% de uso en el sector industrial como técnicas de aplicación principales, mientras que SMED representa el 5,41\% mayormente para el sector automotriz, con un 2,70\% empatan los sistemas Poka-Yoke y Control Visual y, finalmente, las herramientas de Células de Manufactura, Heijunka y Andon con un 1,35\%.

Se encuentran investigaciones en las que se aplican las herramientas de ME con metodologías como Seis Sigma, Simulación, Planificación de los Materiales (MRP), Gestión de la Calidad Total (TQM) y Modelos de Ecuaciones Estructurales teniendo un porcentaje de combinación del 23,53\%, 17,65\%, 29,41\%, 11,76\% y $5,88 \%$, respectivamente. Por el contrario, se observa que la $\mathrm{ME}$ tiene 43,24\% de integración con las herramientas de esta misma en procesos industriales para la mejora continua.

Por medio de esta revisión se percibe un panorama actual en la aplicación e integración de las herramientas que componen la Manufactura Esbelta, buscando las áreas de oportunidad para lograr que el uso de estas sea de forma unificada, por lo cual se obtendría el paquete de beneficios en conjunto.

Es importante tener presente que con los avances tecnológicos y la diversificación de la producción las industrias deben ser flexibles y adaptarse a la demanda de los clientes asegurando la calidad; por lo tanto, el poner énfasis en los sistemas esbeltos es garantizar la satisfacción en los procesos y productos o servicios dados. 
1. Womack JP, Jones DT, Roos D. The Machine that Changed the World: The Story of Lean Production. New York: Harper Collins; 1990.

2. Ohno T. El sistema de producción Toyota: Más allá de la producción a gran escala. 3a ed. Barcelona: Gestión 2000; 1991.

3. Plenert G. What are the Technical Tools of Lean Management. Burlington: Elsevier Incorporated; 2007.

4. Vilana J. Fundamentos del Lean Manufacturing. Madrid: Escuela de Organización Industrial; 2011.

5. Slack N, Lewis M. Operations Strategy: What is Operations Strategy. 2nd ed. Harlow: Prentice Hall/Financial Times; 2008.

6. Marmolejo N, Mejía A. Desarrollo e implementación de un plan de acción de mejora continua mediante las herramientas de la manufactura esbelta en el área de importado de la empresa Colfactory S.A. [tesis]. Cali: Universidad San Buenaventura Cali; 2012.

7. Kahneman D. Thinking, Fast and Slow. New York: Farrar, Straus and Giroux; 2011.

8. Womack J, Jones D. Lean Thinking: Banish Waste and Create Wealth in Your Corporation. New York: Simon \& Schuster; 1996.

9. Shingo S. El sistema de producción Toyota desde el punto de vista de la ingenieria. 3a ed. Madrid: Tecnologías de Gerencia y Producción; 1993.

10. Liker JK. The Toyota way: 14 management principles from the world's greatest manufacturer. New York: McGraw-Hill; 2004.

11. Hossain M., Uddin M. An Approach to Improve the Process Cycle Efficiency and Reduce the Lead Time of a Mango Juice Processing Line by Using Lean Tools: A Case Study. IJSER. 2015; 6(1):1442-1452.

12. Arrieta K. Diseño de una metodología que relaciona las técnicas de manufactura esbelta con la gestión de la innovación: Una investigación en el sector de confecciones de Cartagena (Colombia). Universidad y Empresa. 2015; 17(28):127-145.

13. Dirgo R. Look Forward Beyond Lean and Six Sigma: A Self-perpetuating Enterprise Improvement Method. 2nd ed. Fort Lauderdale: J. Ross Publishing; 2006.

14. Wilches MJ, Cabarcas JC, Lucuara J, Gonzalez R. Aplicación de herramientas de manufactura esbelta para el mejoramiento de la cadena de valor de una línea de producción de sillas para oficina. Dimens Empres. 2013; 11(1):126136.

15. Hirano H. 5 pillars of the visual workplace: the source book for 5 S implementation. 2nd ed. Portland: Productivity Press; 1996.

16. Womack J, Jones, D. Beyond Toyota: How to Root Out Waste and Pursue Perfection. Harvard Bus Rev.1996;75(5):140-158.

17. Vinodha $S$, Vasanth $K$, Vimal K. Implementing lean sigma in an Indian rotary switches manufacturing organization. Prod Plan Control. 2014; 25(4):288302.

18. Valencia S. La filosofía LEAN aplicada en la Gerencia de proyectos. Medellín: Universidad Nacional de Colombia; 2013.

19. Pérez J, La Rotta $D$, Sánchez $K$, Madera $Y$, Restrepo $G$, Rodríguez $M$. Identificación y caracterización de mudas de transporte, procesos, movimientos y tiempos de espera en nueve pymes manufactureras incorporando la perspectiva del nivel operativo. Rev Chil Ing. 2011; 19(3):396-408.

20. Mehta RK, Mehta D, Mehta NK. An Exploratory Study on Implementation of Lean Manufacturing Practices (with Special Reference to Automobile Sector Industry). Yönetim ve Ekonomi. 2012; 19(2):289-299.

21. Gopinath $S$, Freiheit $T$. A waste relationship model and center point tracking metric. IIE Trans. 2011; 44(2):136-154.

22. Juthamas C, Monsiri O, Phrompong S. Improving the productivity of sheet metal stamping subassembly area using the application of lean manufacturing principles. Procedia Manufacturing. 2015; 2:102-107.

23. Spear SJ, Bowen HK. Decoding the DNA of the Toyota production system. Harvard Bus Rev. 1999; 77(5):96-108.

24. Indrawati S, Ridwansyah M. Manufacturing Continuous Improvement Using Lean Six Sigma: An Iron Ores Industry Case Application. Procedia Manufacturing. 2015; 4:528-534.
25. Markovitz D. Information 5S [Web site]. [USA]: Markovitz Consulting; 2017 [cited oct 2017]. Available from: http://www.markovitzconsulting. com/\#ideas

26. Liker JK, Morgan JM. The Toyota product development system. Integrating people, process, and technology. New York: Productivity Press; 2006.

27. Ford H, Crowther S. My Life and Work. New York: Doubleday, Page \& Company; 1922.

28. Ford H, Crowther S. Today and Tomorrow. New York: Doubleday, Page \& Company; 1926.

29. Muhammad F, Syed Asad A, Muhammad A, Muhammad Z, Muhammad M. Energy Management in a Manufacturing Industry through Layout Design. Procedia Manufacturing. 2017; 8(1):168-174.

30. Mehta P, Butkewitsch-Choze S, Seaman C. Data analytics framework for semicontinuous manufacturing process -Implementation vision with a use case. J Manuf Syst. 2017; 44(2):273-279.

31. Stefan $S_{1}$ Jörg B, Joachim M, Amin B. Evaluation of Work Measurement Concepts for a Cellular Manufacturing Reference Line to enable Low Cost Automation for Lean Machining. Procedia CIRP. 2014; 17(1):588-593.

32. Belekoukias I, Garza J, Kumar V. The impact of lean methods and tools on the operational performance of manufacturing organizations. Int J Prod Res. 2014; 52(18):5346-5366.

33. Montilla CA, Gallego JA. Diseño de un semiautomatismo para asistir el proceso de fabricación tijera 45D de motocicleta Yamaha, en la empresa Solomoflex. Scientia et Technica. 2013;18(4):657-663.

34. Bodek N. Jidoka simple tool a complex problem. Morro Bay: Strategies Group LLC; 2011.

35. Shingo S. Zero Quality: Source Inspection and the Poka-Yoke system. Stanford: Productivity Press; 1986.

36. The Lean Enterprise Institute. Lean Lexicon: A Graphical Glossary for Lean Thinkers. 4th ed. Cambridge: The Lean Enterprise Institute; 2008.

37. Meyer, K. Lean Manufacturing history and timeline. Morro Bay: Kanso Strategies; 2011.

38. Kato I, Art S. Toyota Kaizen methods: Six steps to improvement. New York: Productivity Press; 2010

39. Liker J, Rother M. Why lean programs fail [on line]. Cambridge: Lean Enterprise Institute; 2011-2013 [cited 2017 Mar]. Available from: https://www.lean.org/ Search/Documents/352.pdf.

40. Maurer, R. The spirit of Kaizen creating lasting excellence on small step at a time. New York: McGraw-Hill Education; 2012.

41. Imai M. Gemba Kaizen:A Commonsense Approach to a Continuous Improvement Strategy. 2nd ed. Pennsylvania: McGraw-Hill Professional Publishing; 2012.

42. Nor AR, Sariwati MS, Mashitah ME. Lean Manufacturing Case Study with Kanban System Implementation. Procedia Econ Financ. 2013; 7:174-180.

43. Cuatrecasas L. Gestión de la producción, Modelos Lean Management. 2nd ed. Madrid: Diaz de Santos; 2012.

44. Monden Y. Toyota Production System: An Integrated Approach to Just-In-Time. 4th ed. Boca Raton: Taylor \& Francis; 2012.

45. Parthanadee $P$, Buddhakulsomsiri J. Production efficiency improvement in batch production system using value stream mapping and simulation: a case study of the roasted and ground coffee industry. Prod Plan Control. 2014; 25(5):425446.

46. Cusumano M. The Japanese automobile industry: Technology and management at Nissan and Toyota. Cambridge: Harvard University Press; 1985.

47. Shah R, Ward P. Lean Manufacturing: context, practice bundles, and performance. J Oper Manag. 2003; 21(2):129-149.

48. Martínez P, Martínez J, Nuño P, Cavazos J. Mejora en el Tiempo de Atención al Paciente en una Unidad de Urgencias Mediante la Aplicación de Manufactura Esbelta. Inf tecnol. 2015; 26(6):14-40.

49. Espin F. Técnica SMED. Reducción del tiempo de preparación. 3C Tecnología. 2013; 22(1):1-11.

50. Shingo S. Una revolución en la producción: el sistema SMED. 3a ed. Madrid: Taylor \& Francis; 1990. 


\section{REFERENCIAS}

51. Moreira A, Garcez P. Implementation of the single minute Exchange of die (SMED) methodology in small to medium-sized enterprises: A Portuguese casa study. Int J Manag. 2013; 30(1):66-87.

52. Hopp W, Spearman M. Factory physics. New York: McGraw-Hill; 2000.

53. Porras JE. Propuesta para el mejoramiento del proceso productivo de fragancias a través de técnicas LEAN en Disaromas S.A. [tesis]. Granada: Universidad Militar Nueva Granada; 2015.

54. Rahman CML, Hoque MA, Uddin SM. Assessment of total productive maintenance implementation in a semiautomated manufacturing company through downtime and mean downtime analysis. s (Case study: a Semiautomated Manufacturing Company of Bangladesh). IOSRJEN. 2014; 4(9):38-47.

55. Kaizen K. Focused equipment improvement for TPM teams. London: Productivity Press; 1997.

56. Suzuki T. TPM in Process Industries. Portland: Productivity Press; 1994.

57. Mwanza BG, Mbohwa C. Design of a Total Productive Maintenance Model for Effective Implementation: Case Study of a Chemical Manufacturing Company. Procedia Manufacturing. 2015; 4:461-470.

58. Arslankayaa $\mathrm{S}$, Atayb H. Maintenance management and lean manufacturing practices in a firm which produces dairy products. Procedia Soc Behav Sci. 2015; 207:214-224.

59. Rother M, Shook J. Learning to See: value-stream mapping to create value and eliminate muda. 4th. ed. Cambridge: Lean Enterprise Institute; 2009.
60. Cabrera RC. VSM. Value Stream Map. Análisis de la cadena de valor [Blog]. [Trujillo]: Wordpress; 2013 [citado 20 mar 2017]. Disponible en: http://eddymercado. files.wordpress.com/2013/05/analisis-del-mapeo-de-la-cadenade-valor.pdf.

61. Zahraee S, Hashemi A, Abdi A, Shahpanah A, Rohani J. Lean Manufacturing Implementation Through Value Stream Mapping: A Case Study. Jurnal Teknologi. 2014; 68(3):119-124.

62. Tanco M, Santos J, Rodríguez J, Reich J. Applying lean techniques to nougat fabrication: a seasonal case study. Int J Adv Manuf Technol. 2013; 68(1):1639-1654.

63. Engelund EH, Breum G, Friis A. Optimization of large-scale food production using Lean Manufacturing principles. J Foodserv. 2009; 20(1):4-14.

64. Verma N, Sharma V. Energy Value Stream Mapping a Tool to develop Green Manufacturing. (International Conference on Manufacturing Engineering and Materials. ICMEM 2016, 6-10 June 2016; Nový Smokovec, Slovakia). Procedia Engineering. 2016; 149:526-534.

65. Jiménez E, Tejeda A, Pérez M, Blanco J, Martínez E. Applicability of lean production with VSM to the Rioja wine sector. Int J Prod Res. 2012; 50(7):1890-1904.

66. Wang Z, Subramanian N, Gunasekaran A, D.Abdulrahman M, Liu C. Composite sustainable manufacturing practice and performance framework: Chinese autoparts suppliers' perspective. Int J Prod Econ. 2015; 170(1):219-233.

67. Rivera L. Justificación conceptual de un modelo de implementación de Lean Manufacturing. Heuristica 15. 2013; 15(1):91-106. 